\title{
Quantifying proliferative and surface marker heterogeneity in colony-founding connective tissue progenitors and their progeny using time-lapse microscopy
}

\author{
Edward Kwee $^{1,2}$ (D) | Gerald Saidel ${ }^{1}$ (D) | Kimerly Powell ${ }^{3}$ | Christopher Heylman ${ }^{1,2}$ | \\ Cynthia Boehm ${ }^{2}$ । George Muschler ${ }^{2}$ (D)
}

\author{
${ }^{1}$ Department of Biomedical Engineering, Case \\ Western Reserve University, Cleveland, Ohio, \\ USA \\ ${ }^{2}$ Department of Biomedical Engineering, \\ Cleveland Clinic, Cleveland, Ohio, USA \\ ${ }^{3}$ Department of Biomedical Informatics, The \\ Ohio State University, Columbus, Ohio, USA \\ Correspondence \\ George Muschler, Department of Biomedical \\ Engineering, Cleveland Clinic, 9500 Euclid \\ Avenue, Cleveland, $\mathrm{OH} 44195$. \\ Email: muschlg@ccf.org \\ Funding information \\ National Institute of Arthritis and Musculo- \\ skeletal and Skin Diseases, Grant/Award \\ Number: R21 AR067357
}

\begin{abstract}
Connective tissue progenitors (CTPs) are defined as the heterogeneous population of tissue-resident stem and progenitor cells that are capable of proliferating and differentiating into connective tissue phenotypes. The prevalence and variation in clonal progeny of CTPs can be characterized using a colony formation assay. However, colony assays do not directly assess the characteristics of the colony-founding CTP. We performed large, field-of-view, time-lapse microscopy to manually track colonies back to the founding cells. Image processing and analysis was used to characterize the colonies and their founding cells. We found that the traditional colony-forming unit (CFU) assay underestimates the number of founding cells as colonies can be formed by more than one founding cell. After 6 days in culture, colonies do not completely express CD73, CD90, and CD105. Heterogeneity in colony cells was characterized by two cell populations, proliferative and spread cells. Regression modelling of duration of lag phase and doubling time by cell marker suggests the presence of CD90 and CD105 in CTP subpopulations with different proliferative capabilities. From mathematical modelling of clonal colonies, we quantitatively characterized proliferation, migration, and cell marker expression rates to identify desirable clones for selection. Direct assessment of colony formation parameters led to more accurate assessment of CFU heterogeneity. Furthermore, these parameters can be used to quantify the diversity and hierarchy of stem and progenitor cells from a cell source or tissue for tissue engineering applications.
\end{abstract}

\section{KEYWORDS}

cell-surface marker characterization, colony-forming unit assay, quantitative image analysis, stem and progenitor cells, stem cell modelling, time-lapse microscopy

\section{1 | INTRODUCTION}

Cell-based regenerative therapy requires activity of stem and progenitor cells for tissue regeneration. Connective tissue progenitors (CTPs) are tissue-resident stem and progenitor cells that can differentiate into connective tissue phenotypes (Muschler \& Midura, 2002). Cell populations containing cells with CTP properties can be harvested from tissues including bone, bone marrow, and fat. The number of CTPs in a cell source can be estimated using a colony-forming unit (CFU) assay. In the CFU assay, colony-founding CTPs attach to a surface, proliferate, and give rise to a colony of CTP-derived progenitor cells (Friedenstein, Chailakhjan, \& Lalykina, 1970; Pochampally, 2008). Counting the number of colonies allows estimation of the prevalence of CTPs in the starting sample. 
The heterogeneity among colony-founding CTPs can be assessed by measuring differences between colonies (e.g., cell number, cell density, and expression of surface markers). The CFU assay for assessing heterogeneity of CTP and other stem cell types has been standardized with automated CFU analysis (American Society for Testing and Materials F2944-12, 2012). Automated CFU analysis can minimize the high variability in colony counting by subjective reviewers and provides a rapid, objective method to assess functional differences in CTPs and their progeny. Although useful, the CFU assay is limited in that it does not directly assess the colony-founding CTPs. The number and biological variation among clones of colony-founding CTPs are only inferred from the colonies that they form.

It is therefore desirable to design methods that can directly characterize the colony-founding CTP. Clonal analysis can be performed using several established methods, but each are faced with limitations (Hope \& Bhatia, 2011). A variation on the CFU assay that uses limiting dilution to clonally isolate CTPs is challenging because freshly isolated CTPs represent only a small fraction of cells in native tissue. This decreases, but does not eliminate, the chance that a colony has been founded by more than one cell. Establishing a single plating density for the limited dilution approach across multiple donor samples is also confounded by large variation in CTP prevalence between patient samples (Muschler, Nitto, Boehm, \& Easley, 2001). Physical methods to separate cells, such as fluorescence activated cell sorting, can also damage cells and result in a loss of colony-forming efficiency (Mollet, Godoy-Silva, Berdugo, \& Chalmers, 2008).

To overcome the limitations of these methods and enable direct assessment of colony-founding CTPs, we developed a large field-ofview live-cell imaging system with phase-contrast and fluorescence capabilities that enables cell tracking from the time of seeding through colony formation. Using both manual cell tracking and mathematical modelling, quantitative characterization of CTP heterogeneity was developed on the basis of proliferation rate, time to first division, migration rate, and cell marker expression rate of both the founding CTP and progeny. These features derived from time-lapse imaging can be reflective of the identity, starting state, and biological potential of the founding cell. These criteria can be used to understand the diversity and hierarchy of stem and progenitor cells within a cell source and evaluate clonal populations in colonies for subsequent use in research or therapeutic applications.

\section{2 | METHODS}

\section{1 | Time-lapse imaging strategy}

Time-lapse imaging was performed on cells harvested from the trabecular surface of discarded bone core samples. Cells were plated and imaged every hour with phase-contrast microscopy to document colony formation. Cells were labelled every $24 \mathrm{hr}$ with fluorescent antibodies for cell-surface markers to track cell marker expression. Following 6 days in culture, image processing and analysis was performed to segment and characterize colonies. The time lapse was reversed to identify the colony-founding CTP and whether the colony was clonally founded by a single cell. Proliferative cells for both clonal and nonclonal colonies were manually tracked to determine time to first division and doubling time. Clonal colony time-lapse images were processed and analysed with a mathematical model to additionally characterize cell migration rate and surface marker expression rate.

\section{2 | Live-cell imaging system}

A custom, live-cell imaging system was developed for tracking cell proliferation using phase-contrast imaging and changes in cell marker expression using fluorescence imaging. This system uses a DMI6000 inverted fluorescence microscope (Leica Microsystems, Wetzlar, Germany), EL6000 metal halide fluorescence source (Leica), Proscan H117 motorized stage (Prior Scientific, Rockland, MA), and Retiga 2000R camera (QImaging, Surrey, BC, Canada). Fluorescence cubes were selected for imaging green (L5, 480-nm excitation [ex]/527-nm emission [em]), red (TX2, 560-nm ex/645-nm em), and deep red (Y5, $620-\mathrm{nm}$ ex/700-nm em) wavelengths. Automated image acquisition was performed using an OASIS-blue controller card and Surveyor software (Objective Imaging, Cambridge, UK). The imaging system was contained within an Xvivo System (Biospherix, Lacona, NY), which enabled cell culture under controlled oxygen tension.

\section{3 | Cell harvest and culture}

Discarded bone core samples from the proximal femur were received from three patients undergoing hip arthroplasty procedures. Discarded bone core samples from primary hip arthroplasty procedures have been previously studied as a potential stem cell source (Chang, Docheva, Knothe, \& Knothe Tate, 2014; Siclari et al., 2013). Discarded samples were de-identified, human subject research exempt, and acquired under a protocol approved by the Cleveland Clinic Central Biorepository. Samples were sterilely minced in media into 1- to 2-mm fragments using forceps and a sharp osteotome. Medium containing $100 \mathrm{U} / \mathrm{ml}$ Collagenase Type I in Hank's buffered salt solution was added to the fragments of trabecular bone for $1.5 \mathrm{hr}$ at $37^{\circ} \mathrm{C}$. Collagenase activity was stopped using complete aMEM media and $10 \%$ fetal bovine serum at a 1:1 ratio. Cells were passed through a 70- $\mu \mathrm{m}$ cell strainer to filter out bone fragments and large debris. Cells were pelleted and resuspended in complete media to remove excess collagenase. Resulting cells were collected as the trabecular surface cell population (Qadan et al., 2018).

\subsection{Cell culture and time lapse}

Cells were seeded in $2 \times 2-\mathrm{cm} \mu$-Slide $2 \mathrm{Well} \mathrm{Ph}+$ chamber slide (Ibidi, Planegg/Martinsried, Germany) at a plating density of 250,000 cells per chamber in a-MEM media, $10 \%$ fetal bovine serum, and $1 \%$ penicillin-streptomycin. Cells were cultured at 3\% oxygen tension, physiologically relevant conditions for bone-derived CTP characterization (Villarruel et al., 2008).

Cells were allowed $24 \mathrm{hr}$ to settle and adhere to the slide. Cells were washed to remove nonadherent cells to allow visualization of the colony-founding cells. Cells were then labelled using antibodies directly conjugated with fluorophores to characterize surface marker 
expression on live colony-founding cells and their progeny. This technique has been used in several imaging applications for live, surface marker detection (Chan et al., 2009; Eilken et al., 2011). Cell markers CD73, CD90, and CD105 were selected for study. The International Society of Cell Therapy defines multipotent, mesenchymal stromal cells (MSCs) such that $95 \%$ of cells in a population have positive marker expression of CD73, CD90, and CD105 (Dominici et al., 2006). Our aim was to determine the prevalence and variation in freshly isolated CTPs from human trabecular surface cells and the variation in emergence of MSC marker expression among the progeny of colony-founding CTPs during colony formation.

Cells were labelled using directly conjugated antibodies Alexa488CD105 (BioLegend, San Diego, CA), PE-CF594-CD73 (BD Biosciences, San Jose, CA), and Alexa647-CD90 (BioLegend). Controls used to titrate antibody concentrations for live labelling of cell-surface markers resulted in a fluorescent antibody ratio of 1:150, which was added directly to the culture media for $30 \mathrm{~min}$ (Figure S1). Phasecontrast images were taken every hour at $10 \times$ magnification with $2 \times 2$ binning to track cell proliferation and migration. Cells were labelled and fluorescently imaged every $24 \mathrm{hr}$ to detect cell marker expression on the colony-founding cells and their progeny. Prior to fluorescence imaging, a-MEM media were removed. Cells were washed once with phosphate-buffered saline, and OptiKlear Live Cell Imaging Buffer (Marker Gene Technologies, Eugene, OR) was added to reduce background autofluorescence. Cells were cultured for 6 days to provide sufficient time for colonies to develop and minimize the confounding effects of overgrowth or overlap between adjacent colonies.

\section{5 | Image processing and analysis}

Phase-contrast images were processed using custom scripts developed in MATLAB 7.12.0 (MathWorks, Natick, MA). Images were cropped to only include the region of the chamber-containing colonies. Images were subsampled by $50 \%$ for a resolution of $2.7 \mu \mathrm{m}$ per pixel for analysis. Background correction for large dark and bright background artefacts was performed by subtracting a median-filtered $(11 \times 11$ pixel $)$ image from the original image. An $11 \times 11$-pixel bottom-hat filtering was performed on the background-corrected image to filter out bright objects from dark adherent cells. Images were manually threshold segmented, and noncell artefacts were excluded by morphological filtering based on roundness ( $4 \times$ Area/ $\left(\pi \times\right.$ Major Axis Diameter $\left.\left.{ }^{2}\right)\right)$ less than 0.7 and aspect ratio (Major Axis Diameter/Minor Axis Diameter) larger than 1.3 to segment cells with adherent cell morphology. Colonies were identified in Day 6 images to cluster segmented cells using a Euclidean distance of $108 \mu \mathrm{m}$.

Fluorescence images were processed sequentially and analysed using Fiji (Schindelin et al., 2012). Denoising using the Rudin-Osher-Fatemi method was used to reduce background noise in each fluorescence channel. A 21-pixel rolling ball algorithm was used to correct for background fluorescence. Positive cell marker expression was identified using a normalized histogram of the corrected fluorescence image and the triangle threshold algorithm.
Due to the halo effect, phase-contrast images were found to undersegment the cell area relative to the fluorescence segmentation of certain markers. Therefore, total cell area in the colony was calculated as the union of the phase-contrast segmentation and the fluorescence segmentation masks (derived above from MATLAB and Fiji) of all the markers. Analysis of cell, cell marker, and colony segmentation was performed using Fiji. Per cent cell marker expression was normalized to the total cell area. Further details and image workflow are described in Appendix A.

\section{6 | Cell tracking}

Image analysis was performed for colonies at Day 6 (Figure 1a). Following colony identification, after 6 days in culture, the time lapse was reversed, and colonies were manually tracked back to the colonyfounding CTP. Image analysis was performed on CTPs as described above (Figure 1b). Apparent prevalence is calculated by assuming each colony is founded by a single founding cell and dividing the number of colonies identified in the chamber by the total number of cells plated. Colonies founded by a single founding cell were classified as a clonal colony. Colonies founded by more than one cell or were formed by the merging of more than one colony were classified as nonclonal. Colonies not formed by identifiable founding cells and arose as a result of migration from a main colony or displacement from a main colony after a media change were classified as pseudocolonies (Figure 2).

The number of CTPs contributing to colony formation was determined from the cell tracking. This number was used to calculate the observed prevalence of CTPs by dividing the number of cells contributing to colony formation by the total number of cells plated. CTP lag time and doubling time were determined through manual tracking of cell populations. Linear regression analysis was used to determine differences in lag time and doubling time based on CTP cell marker characteristics at Day 1. A Kruskal-Wallis test with multiple comparisons was used to determine differences in lag time and doubling time between patients. Statistical analysis was performed using MATLAB.

\section{7 | Mathematical model for clonal colony characterization}

Clonal colonies were identified, and colony time-lapse images were segmented for the colony formation model. The following processes were modelled: proliferation, cell marker expression, and migration. We assumed that these processes were not limited by the surrounding medium and that cell death was negligible over the experimental period of study. Furthermore, we assumed that random migration can be represented as radially symmetric on a culture surface with no directional impediments.

When performing phase-contrast imaging, cells were often touching, making them difficult to segment into individual cells. Experimentally, it was possible to follow the colony development by measurement of cell area rather than cell number. Assuming the area that cells occupy is proportional to the cell number, a proliferation model can be expressed in terms of the total cell area $A(t)$. A proliferation model assumed with a constant rate coefficient $k_{p}$ after a time 

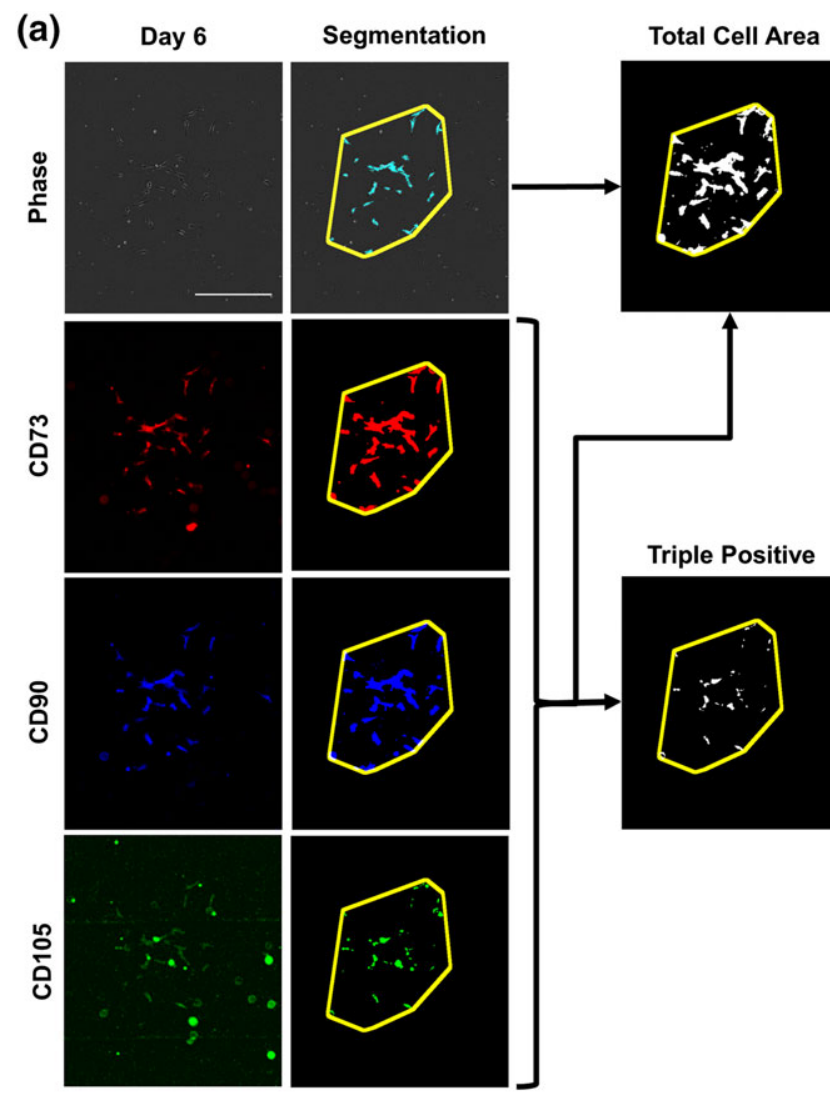

Colony Area
Total Cell Area

Cell Density

CD73 (Area/Percent)

CD90 (Area/Percent)

CD105 (Area/Percent)

Triple Positive (Area/Percent)

\section{Colony Metrics}

$560000 \mathrm{~m}^{2}$

$89800 \mu \mathrm{m}^{2}$

$16.0 \%$

$74000 \mu \mathrm{m}^{2} / 82.4 \%$

$71000 \mu \mathrm{m}^{2} / 78.6 \%$

$15000 \mu \mathrm{m}^{2} / 16.7 \%$

$15000 \mu \mathrm{m}^{2} / 16.7 \%$
$11400 \mu \mathrm{m}^{2} / 12.7 \%$

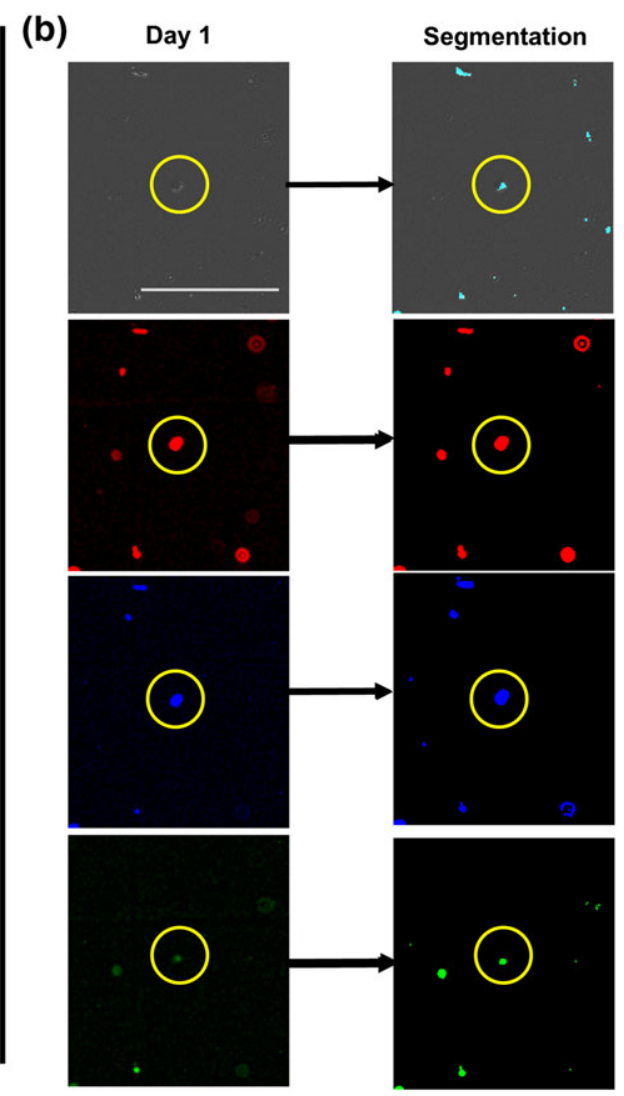

\begin{tabular}{ll}
\multicolumn{2}{c}{ CTP Metrics } \\
Cell Area & $430 \mu \mathrm{m}^{2}$ \\
CD73 & $2200 \mu \mathrm{m}^{2}$ \\
CD90 & $2200 \mu \mathrm{m}^{2}$ \\
CD105 & $550 \mu \mathrm{m}^{2}$
\end{tabular}

FIGURE 1 Representative colony analysis. (a) Cells were segmented in phase (white) and clustered to identify colonies (yellow). Cell markers were segmented, and area expression within the colony was determined. Triple positive area for CD73, CD90, and CD105 was determined as the common area between the three markers. (b) Colony was traced back to the colony-founding cell (yellow). Phase-contrast segmentation (cyan) and fluorescence segmentation were used to determine connective tissue progenitor (CTP) metrics. Time lapse in Movie S1. Scale bar: $500 \mu \mathrm{m}$ [Colour figure can be viewed at wileyonlinelibrary.com]

delay $\tau$, which represents the duration of the lag phase. The total cell area at any time is the integral of the average cell area density $a(r, t)$ locally at any radial position over an arbitrarily large radius $\left(r_{\infty}\right)$ on a material surface (Equation 1).

$A(t)=2 \pi \int_{0}^{r_{\infty}} r a(r, t) d r \quad \Rightarrow \quad \frac{d A}{d t}=\begin{aligned} & 0, t<\tau \\ & k_{P} A, t \geq \tau\end{aligned} \quad t=0, \quad A=A^{0}$

where $A^{0}$ is the initial cell area. Assuming that cells move by random migration with coefficient $D$ and proliferate with rate coefficient $k_{P}$, the local area cell number density changes over the spatial domain $0 \leq r \leq r_{\infty}$ according to

$$
\frac{\partial a}{\partial t}=\frac{1 \partial}{r \partial r} r D \frac{\partial a}{\partial r}+k_{p} a
$$

At the initially low cell density, we expect the least hindrance to migration. As the cell population and cell density increase, greater contact inhibition decreases the migration rate, which can be empirically represented as $D\left[a(r, t)=D_{0} e^{-\phi a^{4}}\right.$, where $D_{0}$ is the initial migration rate coefficient and $\phi$ is a contact inhibition constant. Contact inhibition has been previously found to be important in stem cell proliferation (Hoffmann, Kuska, Zscharnack, Loeffler, \& Galle, 2011). For larger values of $a(r, t), D$ decreases quickly from $D_{0}$ to a small value indicated by the gradient:

$$
\frac{d D}{d a}=-4 \phi a^{3} D_{0} \exp -\phi a^{4}=-4 \phi a^{3} D
$$

Combining Equations 2 and 3, the average area density of all cells changes according to

$$
\frac{\partial a}{\partial t}=D\left\{\frac{\partial^{2} a}{\partial r^{2}}+\frac{1}{r} \frac{\partial a}{\partial r}-4 \phi a^{3} \frac{\partial a^{2}}{\partial r}\right\}+k_{p} a
$$

Corresponding to the model of total cell area in a colony, we construct a model of the triple positive surface area expression of CD73, CD90, 

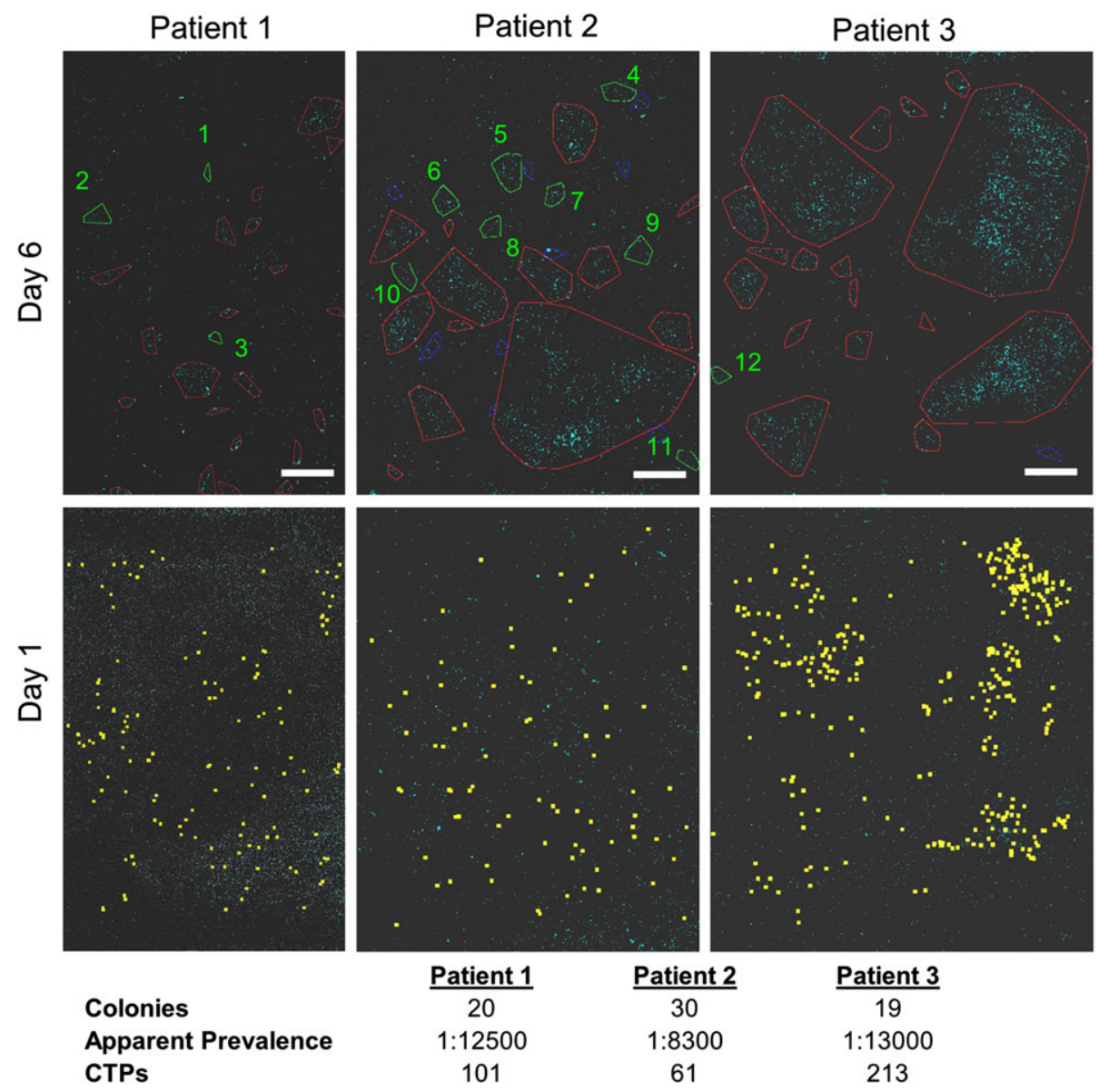

\begin{tabular}{c} 
Patient 1 \\
\hline 20 \\
$1: 12500$ \\
101 \\
$1: 1800$
\end{tabular}

\begin{tabular}{c} 
Patient 2 \\
\hline 30 \\
$1: 8300$ \\
61 \\
$1: 3300$
\end{tabular}

\begin{tabular}{c} 
Patient 3 \\
\hline 19 \\
$1: 13000$ \\
213 \\
$1: 740$
\end{tabular}

FIGURE 2 Colony tracking: Phase-contrast images after 6 days in culture from three different patients were segmented for cells (cyan). Colonies were classified as clonal (green), nonclonal (red), or migratory (blue). Apparent prevalence was calculated on the basis of number of colonies. Tracking the colonies back to the first phase-contrast image enabled identification of cells contributing to colony formation (yellow). Proliferative connective tissue progenitors (CTPs) were identified, and observed prevalence was calculated. Green numbers indicate clonal colony number for modelling. Scale bar: 2,000 $\mu \mathrm{m}$ [Colour figure can be viewed at wileyonlinelibrary.com]

and CD105 at any time. The total area of cells with surface expression, $A_{E}$, corresponding to Equation (1) is

$$
\frac{d A_{E}}{d t}=k_{E} A, \quad t>\tau ; \quad A_{E}=A_{E}^{0}, \quad t \leq \tau
$$

This model assumes negligible proliferation and death of the cells with surface marker expression; however, the increase of cell-surface expression at rate $k_{E}$ is proportional to the area of all cells: $k_{E} A$. Corresponding to Equation (4), the average area density of cells with surface expression, represented by $a_{\mathrm{E}}$, changes with time according to

$$
\frac{\partial a_{E}}{\partial t}=D_{E}\left\{\frac{\partial^{2} a_{E}}{\partial r^{2}}+\frac{1}{r} \frac{\partial a_{E}}{\partial r}-4 \phi a_{E}^{3} \frac{\partial a_{E}}{\partial r}{ }^{2}\right\}+k_{E} a ; \frac{d D_{E}}{d a}=-4 \phi a_{E}^{3} D_{0, E}
$$

where $k_{E} a$ is the rate of area cell-surface expression resulting from nonexpressing cells. Note that the solution to Equation (6) depends on the solution of Equation (4).

The initial conditions were based on the initial area of the colonyfounding cell:

$$
t=\tau: a=a_{0} U\left[r_{0}-r, \quad a_{E}=a_{E, 0} U r_{E, 0}-r\right.
$$

where $U$ is the unit step function, $a_{0}=\pi r_{0}^{2}$, and $a_{E, 0}=\pi r_{E, 0}^{2}$. At the centre of the surface $(r=0)$, the gradients are zero by radial symmetry; at the farthest edge of the surface $\left(r=r_{\infty}\right)$, there are no cells:

$$
r=0: \frac{\partial a}{\partial r}=\frac{\partial a_{E}}{\partial r}=0, \quad r=r_{\infty}: a=a_{E}=0
$$

\section{8 | Model discretization}

Experimentally, corresponding to measurements of cell distribution over discrete spatial regions, we express the model in discretized form. In the continuous spatial domain $0 \leq r \leq r_{\infty}$, we define a discrete spatial domain $i=0,1,2, \ldots, M$, where $M$ is the number of spatial intervals of size $\Delta=r_{\infty} / M$ (Figure 3a). The relationships between continuous and discrete spatial variables are $r_{i}=i \Delta, D_{i}(t)=D_{o} e^{-\phi a_{i}{ }^{4}}, a_{i}(t)=a\left(r_{i}, t\right) d r_{i}$, and $a_{E, i}(t)=a_{E}\left(r_{i}, t\right) d r_{i}$. The fractional area occupied by nonexpressing or expressing cells in the interval is $d r_{i}$. Second-order central difference for the second derivative and a first-order forward difference for the first derivative were implemented. 

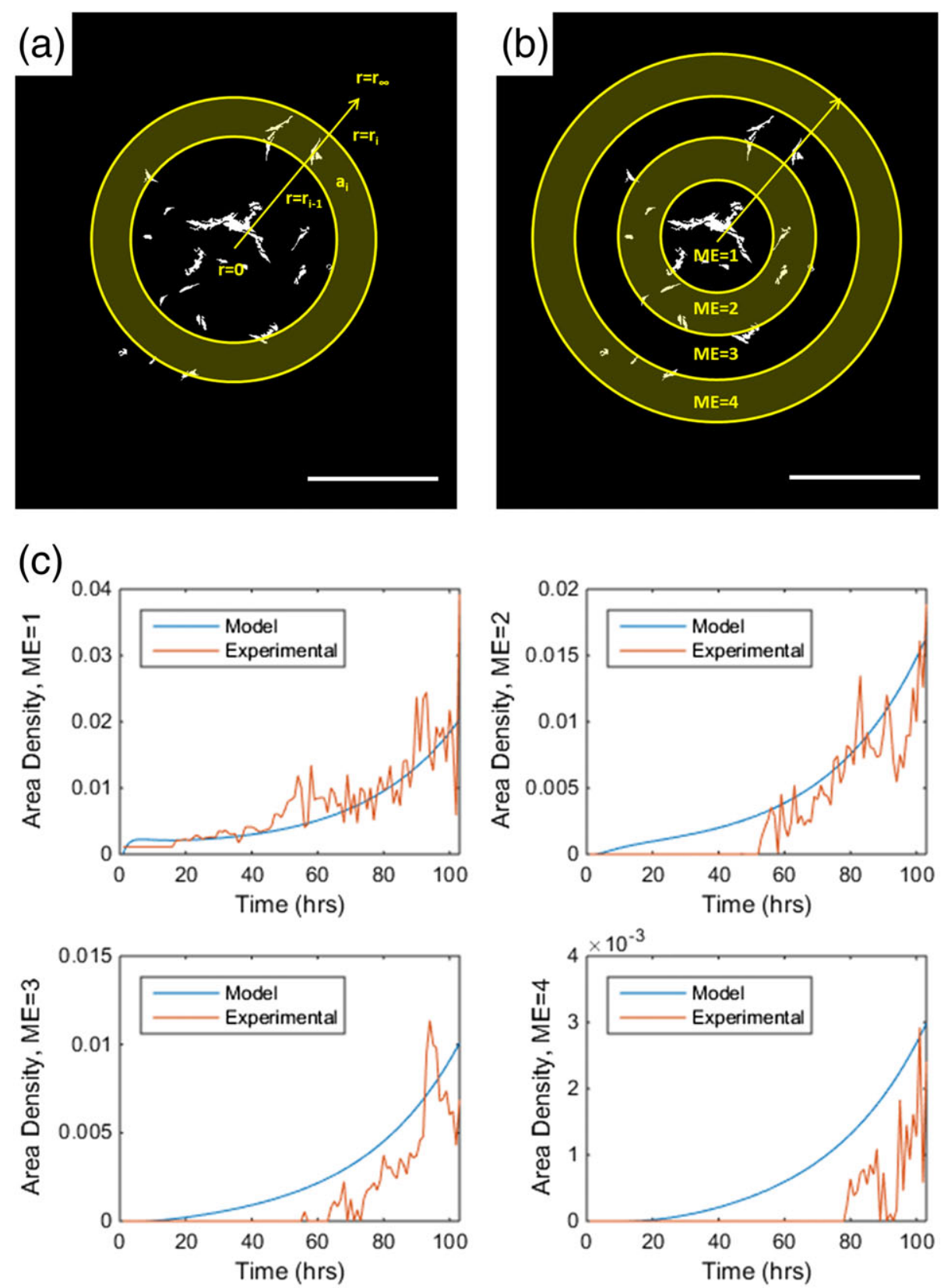

FIGURE 3 Colony formation model. (a) Cell segmentation modelled with a radially symmetric model based on the centroid of the colony. (b) Four experimental intervals $\left(M_{E}\right)$ were used for parameter estimation. (c) Total cell area density plot for a representative colony: Area density is modelled in four experimental intervals $\left(M_{E}\right)$. Best fit parameters were determined with respect to the experimental data. In this example, $D_{0}=1.9 \mathrm{e} 3 \mu \mathrm{m}^{2} / \mathrm{hr}$. Scale bar $500 \mu \mathrm{m}$ [Colour figure can be viewed at wileyonlinelibrary.com]

\section{9 | Model cell segmentation}

Segmented images were discretized by first transforming every segmented cell pixel from Cartesian coordinates to polar coordinates, where the centre of the colony was the origin. The total number of pixels was used as experimental data for the spatially lumped model for cell area. The spatially lumped data from phase-contrast imaging were used to estimate the proliferation constant $k_{p}$ for the total cell area colony with Equation (1). The spatially lumped data from the fluorescence imaging were used to estimate $k_{E}$ for rate of triple positive cell expression with Equation (5).

Cell area density for cells with or without surface expression was discretized into equally spaced (radially symmetric) experimental intervals, $M_{E}$ (Figure 3b). The outer boundary of radial domain was chosen as $r_{\infty}$, the minimum radius at which no cells were present throughout the experiment. The cell area density at $r=0, a_{0}$, is assumed to be equal to $a_{1}$ according to the initial cell area density. The average area fraction was defined in each of the equally spaced intervals $i=1,2, \ldots M$. Because $M_{E}<M$, we obtain an average cell area fraction, which is a partial sum with $\left(M / M_{E}\right)=p$ points for each interval $k$ :

$$
A_{k}=\frac{\sum_{i=(k-1) p+1}^{k p} a_{i} r_{i}}{\sum_{i=(k-1) p+1}^{k p} r_{i}}
$$


In this study, the number of intervals for simulation was $M=100$, and the number of experimental intervals is $M_{E}=4$ so that $p=25$, where

$M_{E}=1, \quad A_{1}=\frac{\sum_{i=1}^{25} a_{i} r_{i}}{\sum_{i=1}^{25} r_{i}} ; M_{E}=2, \quad A_{2}=\frac{\sum_{i=26}^{50} a_{i} r_{i}}{\sum_{i=26}^{50} r_{i}} ; \ldots M_{E}=4, \quad A_{4}=\frac{\sum_{i=76}^{100} a_{i} r_{i}}{\sum_{i=76}^{100} r_{i}}$.

\subsection{0 | Simulation and parameter estimation procedure}

Simulations were performed in MATLAB to simultaneously estimate the migration coefficient $D_{0}$ based on total cell area density discretization and the triple positive migration coefficient $D_{E, O}$ for the discretized positive area density expression. The first time point after $t=\tau$ was discretized into experimental intervals $M$ and used as the initial condition for simulations. Simulations were evaluated with experimental data by averaging model outputs $a_{i}(t)$ and $a_{E, i}(t)$ in intervals.

In these model equations, the model parameters characterize lag time to division, total cell proliferation rate, cell marker expression rate, total cell migration, cell marker proliferation rate, and contact inhibition as indicated by the experimental data of colony formation. A sequential strategy was applied to estimate parameters: (a) proliferation coefficient $k_{p}$ and lag time $\tau$ from $A(t)$ data; (b) total cell migration coefficient $D_{0}$ and $\phi$ from $a_{i}(t)$ data; and (c) triple positive cell expression $k_{E}$ and migration $D_{O, D}$ from $A_{E}(t)$ data. The optimal parameter estimates were obtained by minimizing an objective function defined as the sum of least squares between the simulated and the experimental data for four discretized areas $M_{E}$ and time points (Figure 3c).

Parameters were normalized to their highest respective value for ranked comparison among colonies. From these results, we can expect colonies with the highest normalized values of $k_{P}, D_{0}, k_{E}$, and $D_{O, E}$ and the lowest normalized values of $\tau$ and $\phi$ to characterize CTPs and potential for selection in clinical application. We expect cells with the greatest proliferation, migration, and appropriate cell marker expression to be the desired clones of cells for biomanufacturing.

\section{3 | RESULTS}

\subsection{Colony cell marker expression}

Phase-contrast images taken after 6 days in culture were processed and segmented to automatically identify cells and colonies. Among three patient samples, 69 colonies were identified. The per cent area expression of each cell marker for each colony was determined (Figure 4). Colonies were on average $81.5 \%$ positive for CD73, $52.4 \%$ positive for CD90, $18.8 \%$ positive for CD105, and $11.6 \%$ positive for all three markers.

\section{2 | Colony-founding cell identification and analysis}

After colony identification, the time lapse was reversed to identify the colony-founding cell (sample colony time lapse in Movie S1). This analysis demonstrated that colonies were not always founded by a single cell (Figure 2). Twelve clonal colonies, 47 nonclonal colonies, and 9 pseudocolonies were identified across the three patient samples. CTPs $(n=375)$ with proliferative cell progeny contributed to colony formation (Figure 5). The $97.6 \%(n=366)$ were positive for CD73 and $81.2 \%(n=291)$ for CD90. Only $18.8 \%(n=103)$ of CTPs were positive for CD105. The $97.0 \%$ of the CD105 positive cells were also positive for CD73 and CD90 ( $n=100)$.

CTP progeny proliferation was manually tracked over the course of the time lapse. Time to first division, or the end of the lag phase, was determined. Cell population was also counted to determine a doubling time according to an exponential growth model that fit well with Pearson's correlation coefficient of $0.95 \pm 0.04$ (mean \pm standard deviation). Doubling time for CTPs also varied with an average of $22.5 \pm 8.74 \mathrm{hr}$. Time to first division varied with an average of $75.8 \pm 15.3 \mathrm{hr}$ (Figure 5). Regression modelling was used to determine if there was a correlation between cell marker expression at Day 1 with lag phase duration and doubling time (Table 1). Positive expression of CD90 on the CTP at Day 1 was significantly correlated with higher doubling time. Positive expression of CD105 was significantly correlated with decreased time to the first division.

\section{3 | Mathematical model of colony formation dynamics}

Time-lapse data from 12 clonal colonies were analysed to quantify the characteristics of CTPs. The spatially lumped model for total cells was used to simulate experimental data of the total cell area in the colony and to estimate lag time $\tau$ and proliferation rate $k_{p}$. Using the same lag phase $\tau$ determined from the total cell area data, the model for triple positive cell area simulated the corresponding experimental data in order to estimate the proliferation rate $k_{E}$ for positive expressing cells (Figure 6).

The development of larger colonies was characterized by larger $k_{P}$ or proliferation rates. The degree to which contact inhibition influenced colony migration is indicated by $\phi$, which varied from 0.4 to 4.0. Lag time $\tau$ varied between 25 and $65 \mathrm{hr}$. A comparison of parameter values for each colony showed a positive correlation between $k_{P}$ and $k_{E}$, indicating more proliferative colonies had higher rates of differentiation. A positive correlation was observed between $k_{P}$ and $D_{0}$, indicating more proliferative colonies also had higher migration rates. Lastly, a positive correlation was observed between $k_{E}$ and $D_{O, E}$. This indicated triple positive cells with more proliferation had higher cell migration. A sensitivity analysis was applied to show that colony ranking was insensitive to a range of values for the lag phase and contact inhibition constant (Appendix B).

\section{4 | DISCUSSION}

A system for cell culture, live-cell imaging and analysis was developed for direct identification and quantitative characterization of colonyforming cells under physiological conditions. These included appropriate oxygen tension in a two-dimensional cell culture environment prior to the first cell division in vitro. These techniques enabled direct 


\section{(a) Patient 1}

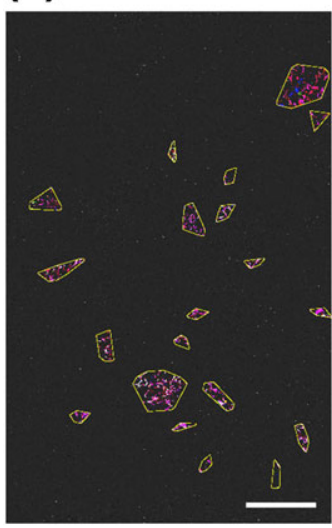

\section{- CD73 -CD90}

Patient 2

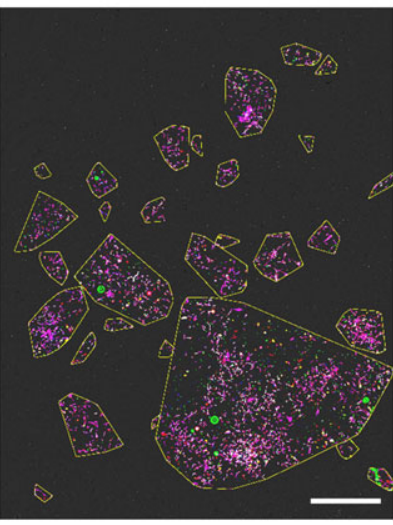

Patient 3

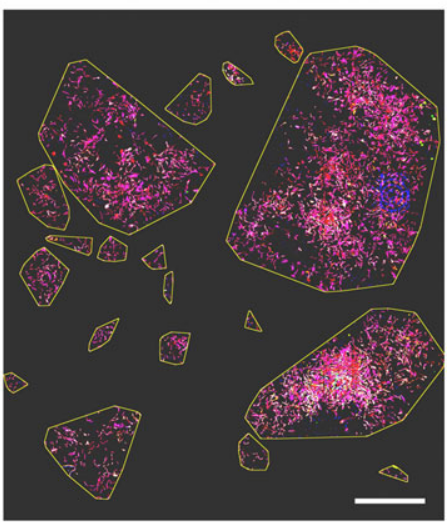

(b)

\section{Colony Percent Cell Surface Marker Expression}

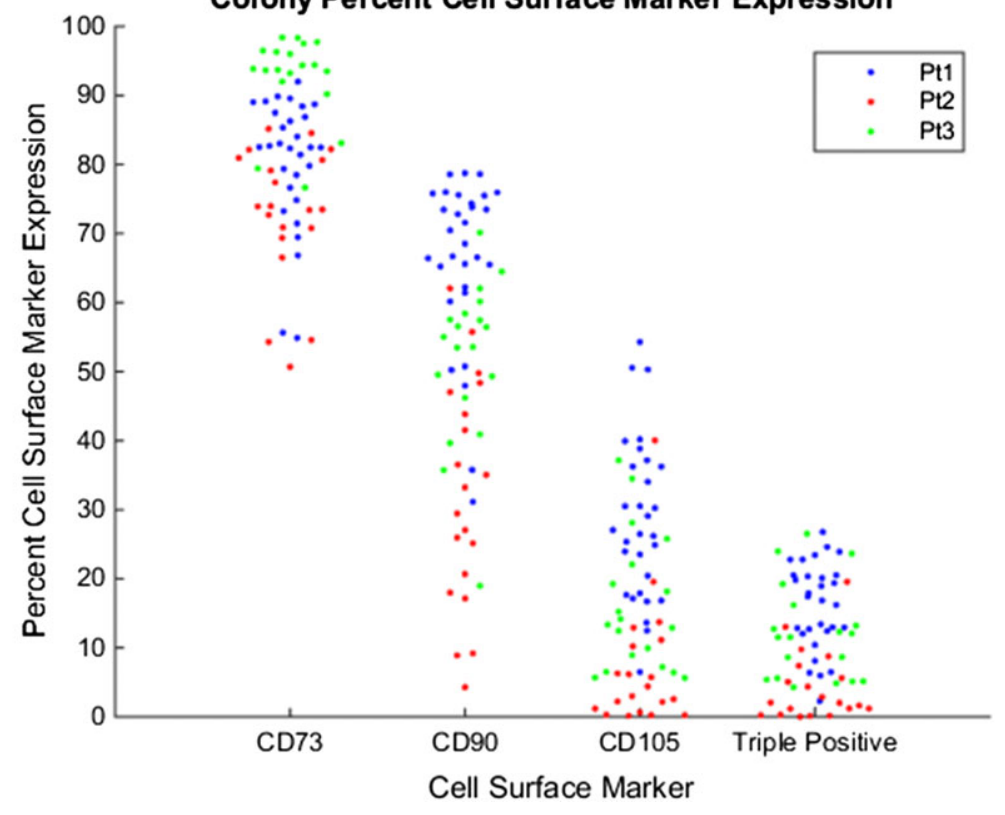

FIGURE 4 Cell marker distribution. (a) Overlay of CD73, CD90, and CD105 expression within colonies. (b) Plot of colony cell marker expression. Each point represents a different colony from a given patient. Cell marker expression per colony was normalized to total cell area in the colony. Scale bar: 2,000 $\mu \mathrm{m}$ [Colour figure can be viewed at wileyonlinelibrary.com]
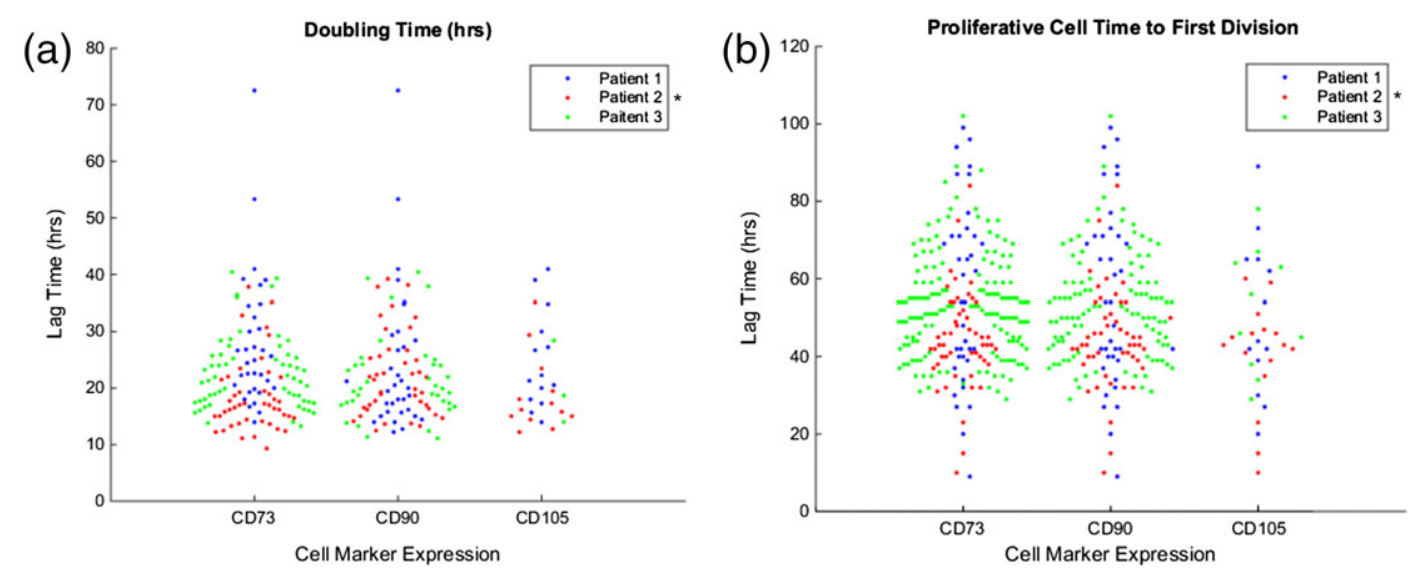

FIGURE 5 Connective tissue progenitor heterogeneity. Connective tissue progenitor proliferative characteristics were quantified over the course of the time lapse according to (a) duration of the lag phase and (b) doubling time. Patient 2 lag time and doubling time were significantly lower than the other patients $\left({ }^{*} p<0.05\right)$ [Colour figure can be viewed at wileyonlinelibrary.com] 
TABLE 1 Linear regression for doubling time and duration of lag phase based on cell marker expression of initial CTP at the start of the time lapse

\begin{tabular}{|c|c|c|c|c|c|c|c|}
\hline \multicolumn{4}{|c|}{ Doubling time regression coefficients } & \multicolumn{4}{|c|}{ Lag phase duration regression coefficients } \\
\hline Term & Coefficient & Standard error coefficient & $p$ value & Term & Coefficient & Standard error coefficient & $p$ value \\
\hline Constant & 18.617 & 6.1849 & 0.003 & Constant & 71.392 & 10.823 & 0 \\
\hline CD90 & 5.7142 & 1.8837 & 0.002 & CD90 & -1.3917 & 2.3245 & 0.549 \\
\hline CD105 & 3.3253 & 1.7867 & 0.065 & CD105 & -5.9021 & 2.6419 & 0.026 \\
\hline
\end{tabular}
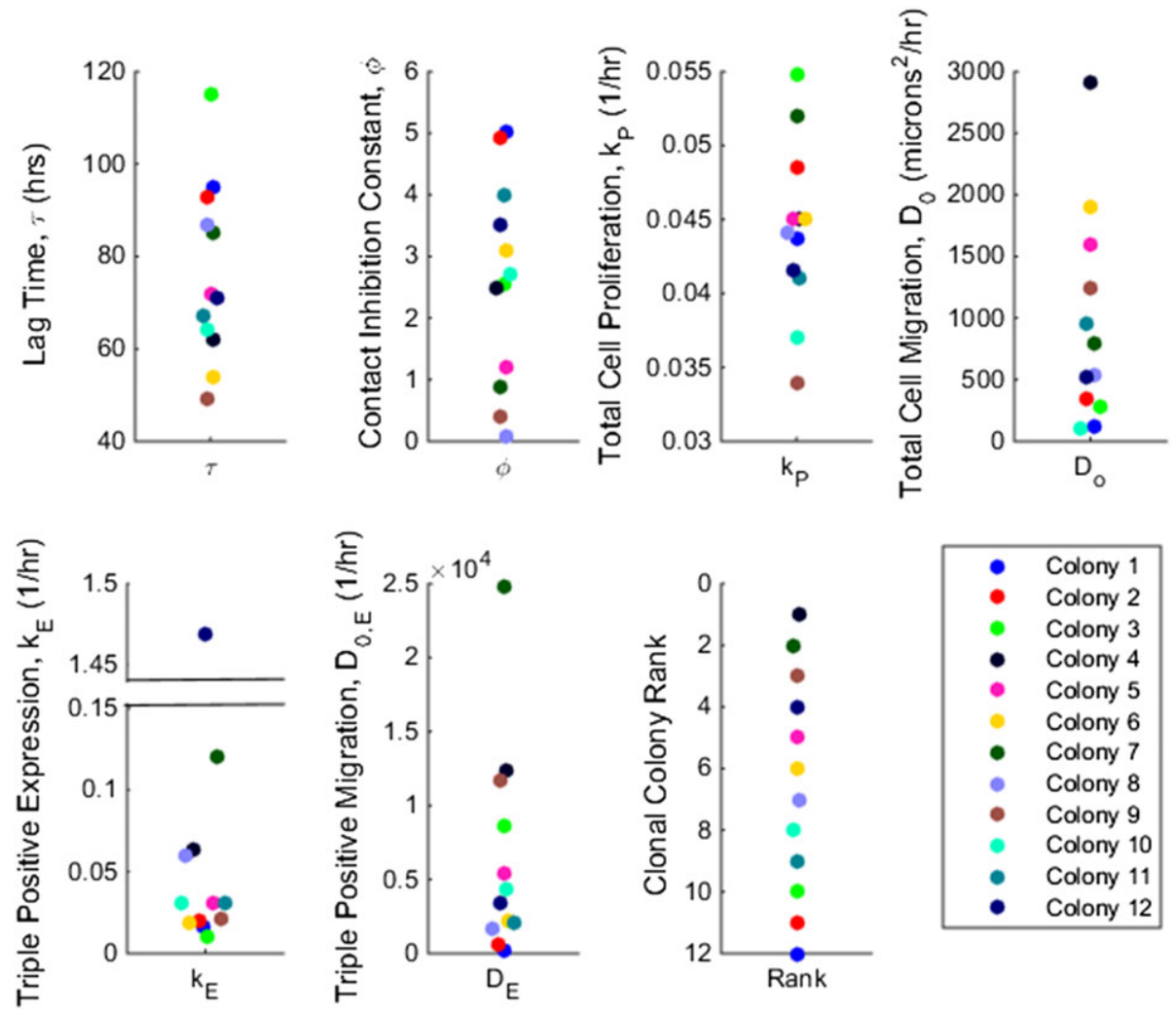

FIGURE 6 Colony model parameters. Model parameters were fit to the experimental data to produce six parameters for colony formation. Equally weighing each parameter enabled assigning of clonal colony ranks, with the highest ranked colony (Rank 1) representing the highest parameters of proliferation and migration for total cells and cell marker expression [Colour figure can be viewed at wileyonlinelibrary.com]

assessment of prevalence, proliferative, and cell marker attributes of colony-founding cells derived from trabecular surface of human cancellous bone.

The prevalence of colony-founding cells is very low in many biological systems. As a result, samples of tissue-derived cells and cells in the early culture environment will contain a greater number of nonprogenitor and nonstem cells than stem and progenitor cells. Selection of stem and progenitor cells can be accomplished by identifying unique surface markers or physical attributes. The systematic and objective study of CTPs from various tissue sources or the design of optimal methods for rapid CTP characterization and isolation requires greater understanding of the attributes of native tissueresident CTPs. This paper contributes both valuable tools and useful information towards this objective. The determination of markers that predict colony formation could be to (a) distinguish CTPs from nonCTPs in freshly isolated cell preparations, (b) define or predict the quality or biological potential of a population of cells obtained from a given donor or tissue, or (c) provide useful diagnostic or therapeutic information related to tissue health or disease.

The methods reported here yield an improved objective assessment of the accuracy of traditional CFU assays. The results suggest that a variety of variables may contribute to underestimation or overestimation of CTP number. We found that CTP prevalence estimated by counting apparent colonies at Day 6 was substantially less than the prevalence measured from the number of colony-founding cells that contribute to those colonies. This occurred when several CTPs attached close enough together on the culture surface such that proliferation and migration resulted in progeny populations combining into one apparent but nonclonal colony.

Identification of individual colony-founding cells enabled the characterization of CTPs with respect to expression of CD73, CD90, and CD105. Examining the cell marker expression on the colony-founding cells, we observed that most cells were positive for CD73 and CD90. Few cells were positive for CD105. As the CTPs proliferated and 
formed colonies, we found that the trend of significant expression for CD73 and CD90 and relatively little expression for CD105 remained consistent. Their progeny do not conform to the positive cell marker criteria set by International Society of Cell Therapy for MSCs. Previous publications show variation in CD105 in early culture and an increase in CD105 expression with more time in culture (Anderson, CarrilloGálvez, García-Pérez, Cobo, \& Martín, 2013; Pittenger et al., 1999; Wang et al., 2014). It is possible that the progeny of CTPs from trabecular surface cells may go on to fully express all three markers at high levels after a longer period of expansion. However, although individual batches of culture expanded MSCs may vary little with respect to expression of CD73, CD90, and CD105, they vary widely with respect to other markers associated with multipotent cell properties (Qadan et al., 2018). As a result, alternative markers may provide more discriminating metrics for prediction of future performance.

Time-lapse microscopy enabled quantitative characterization of two key functional metrics of colony formation: the time lag between plating and cell division for CTPs and the doubling time of the progeny of individual CTPs after first cell division. Although these parameters varied between CTPs, CD90 expression by CTPs was associated with significantly longer doubling time (slower proliferation). CTPs that expressed CD105 at $24 \mathrm{hr}$ demonstrated significantly shorter lag time between plating and first cell division, possibly suggesting that expression of CD105 may increase the chance that the CTP involved is already in the cell cycle and divide. Other studies that show subpopulations of MSCs based on CD90 and CD105 expression may have different osteogenic potential (Pérez-Silos, Camacho-Morales, \& Fuentes-Mera, 2016). However, this was not tested in this study.

The correlation between lag time, proliferation rate, and cellsurface marker expression may provide a functional means of selecting subpopulations of CTPs with preferred biological attributes. All of the CTPs derived in Patient 2 were found to have lag and doubling time significantly lower than those from other patients, reinforcing the concept of patient to patient variation. However, the clinical implications (diagnostic or predictive value) of this variation remains uncertain.

Using the mathematical model, we were able to quantitatively characterize differences between colonies in proliferation rate, lag time, and migration using total cell area and triple positive cell area occupied in discrete radial intervals. Although these parameters can be estimated with conventional imaging, individual cell tracking of centroids, trajectories, and automated lineage mapping over time would be required. Such analysis is problematic when cells are overlapping and touching in phase-contrast images. This method of parameter estimation by modelling cell area growth provides a simpler alternative approach for clonal population characterization.

Colonies with higher cell proliferation rates were associated with higher migration rates. These colonies with faster growth were also associated with higher rate of triple positive area expression. By equally weighing all simulation parameters, colonies were ranked, thereby categorizing specific colonies that could be suitable for picking and selection for further expansion based on a combination of proliferation rate and cell marker expression. Different ranking methodologies can be used depending on the specific needs or release criteria for a cell therapy product.
This model was developed to deal with the limitations of the imaging data. Segmenting single cells with conventional automated thresholding techniques, such as Otsu's method, is not possible due to the halo effect and shade off artefacts in phase-contrast imaging and necessitated the use of a manual threshold. Improved automated segmentation algorithms designed for phase-contrast images can be used to more accurately segment individual cells (Chalfoun et al., 2013; Jaccard, Szita, \& Griffin, 2017). Additionally, quantitative phase imaging technologies, which are free of artefacts and more easily segmented with conventional threshold algorithms, can be utilized here (Zangle \& Teitell, 2014).

Additionally, cells are constantly changing in cell area as they move and divide over the course of the time lapse, which contributed to experimental noise in the area measurements. Model assumptions of radial symmetry can be broken when cells divide, but the natural tendency for cells to migrate away restores radial symmetry in colonies over time.

To evaluate cell migration coefficients, we simulated the cell number density variation with radius. Setting the outer radius of the model could be automated to more easily prevent empty simulation intervals. With sparse data, it was necessary to reduce the number of experimental radial intervals to 4 to get a reasonable estimates of $D_{0}$ and $D_{O, E}$. For cell types that change shape less over time, more experimental intervals could be used to provide better granularity to the output data. Cell marker data were limited by fluorescence cell marker labelling performed every $24 \mathrm{hr}$. More frequent labelling and fluorescence imaging may provide more accurate determination of relevant variation in expression parameters. Longer term time-lapse and modelling studies are needed to determine if early proliferation, migration, and marker expression behaviour can be used to predict and select for preferred biological potential among CTP progeny.

In this study, characterization of colony-founding cells began $24 \mathrm{hr}$ after seeding. Imaging the cells immediately after seeding is compromised by the presence of obscuring nonadherent cells, which must be removed. Therefore, changes in cell marker expression that may have occurred between the time of seeding and $24 \mathrm{hr}$ later were not observed. Previous studies have identified $13 \mathrm{hr}$ as the mean adherence time for MSCs (Schlie, Gruene, Dittmar, \& Chichkov, 2012). An earlier media change and characterization of the colonyfounding cells may better reflect the native state of CTPs.

Future work includes analysing other morphological attributes as well as surface markers that may have predictive value in identifying CTP subtypes with preferred biological attributes in short- and longterm cultures and in tissue-specific differentiation assays. Migration rates determined from mathematical modelling can also serve as a measure of potency. Proliferative colonies with higher migration rates tend to be more chondrogenic than slower proliferating colonies (Bertolo et al., 2015). Non-invasive assessments of cell morphology based on observed differences in culture-expanded MSC populations of different trilineage differentiation capabilities may be informative in inferring colony-level heterogeneity (Seiler et al., 2014). However, trilineage differentiation needs to be linked to early functional metrics and surface markers to enable modelling and selection based on biological potential.

To our knowledge, this is the first application of live-cell, timelapse imaging for quantitative analysis of the colony formation assay. 
Time-lapse imaging and image analysis increased the accuracy of the CFU assay by moving from the apparent prevalence, on the basis of the assumption that one CFU founded every apparent colony, to directly measuring the observed true CFU prevalence. Direct observation provides more definitive documentation of colony-founding cells in the starting cell population and improves estimates of CFU prevalence. Measurement of colony formation parameters as determined by our direct analysis more accurately characterizes CFU heterogeneity. These methods directly enable increased precision in CTPs assays and in examination of early variation in biological performance that may be used to dissect hierarchy in native tissues and in the selection of CTP clones and sources with preferred biological performance for use in cell therapy.

\section{ACKNOWLEDGEMENTS}

Research reported in this publication was supported by the National Institute of Arthritis and Musculoskeletal and Skin Diseases of the National Institutes of Health under Award Number R21 AR067357. The content is solely the responsibility of the authors and does not necessarily represent the official views of the National Institutes of Health.

The authors thank the reviewers for their feedback in improving the quality of the manuscript.

\section{CONFLICT OF INTEREST}

The authors have no conflict of interest regarding the publication of this article.

\section{ORCID}

Edward Kwee (iD https://orcid.org/0000-0001-5460-3913

Gerald Saidel (10) http://orcid.org/0000-0003-2159-2343

George Muschler (D) http://orcid.org/0000-0002-0851-8352

\section{REFERENCES}

Anderson, P., Carrillo-Gálvez, A. B., García-Pérez, A., Cobo, M., \& Martín, F. (2013). CD105 (endoglin)-negative murine mesenchymal stromal cells define a new multipotent subpopulation with distinct differentiation and immunomodulatory capacities. PLoS One, 8(10), e76979. https:// doi.org/10.1371/journal.pone.0076979

Bertolo, A., Gemperli, A., Gruber, M., Gantenbein, B., Baur, M., Pötzel, T., \& Stoyanov, J. (2015). In vitro cell motility as a potential mesenchymal stem cell marker for multipotency. Stem Cells Translational Medicine, 4(1), 84-90. https://doi.org/10.5966/sctm.2014-0156

Chalfoun, J., Kociolek, M., Dima, A., Halter, M., Cardone, A., Peskin, A., .. Brady, M. (2013). Segmenting time-lapse phase contrast images of adjacent NIH 3T3 cells. Journal of Microscopy, 249(1), 41-52. https:// doi.org/10.1111/j.1365-2818.2012.03678.x

Chan, E. M., Ratanasirintrawoot, S., Park, I. H., Manos, P. D., Loh, Y. H., Huo, H., ... Schlaeger, T. M. (2009). Live cell imaging distinguishes bona fide human iPS cells from partially reprogrammed cells. Nature Biotechnology, 27(11), 1033-1037. https://doi.org/10.1038/nbt.1580

Chang, H., Docheva, D., Knothe, U. R., \& Knothe Tate, M. L. (2014). Arthritic periosteal tissue from joint replacement surgery: A novel, autologous source of stem cells. Stem Cells Translational Medicine, 3(3), 308-317. https://doi.org/10.5966/sctm.2013-0056

Dominici, M., Le Blanc, K., Mueller, I., Slaper-Cortenbach, I., Marini, F., Krause, D., ... Horwitz, E. (2006). Minimal criteria for defining multipotent mesenchymal stromal cells. The International Society for Cellular Therapy position statement. Cytotherapy, 8(4), 315-317. https://doi.org/10.1080/14653240600855905
Eilken, H., Rieger, M., Hoppe, P., Hermann, A., Smejkal, B., Drew, E., ... Schroeder, T. (2011). Continuous long-term detection of live cell surface markers by 'in culture' antibody staining. Protocol Exchange. https://doi.org/10.1038/protex.2011.205

American Society for Testing and Materials F2944-12 (2012). Standard test method for automated colony forming unit (CFU) assays-Image acquisition and analysis method for enumerating and characterizing cells and colonies in culture. West Conshohocken, PA: ASTM International.

Friedenstein, A. J., Chailakhjan, R. K., \& Lalykina, K. S. (1970). The development of fibroblast colonies in monolayer cultures of guinea-pig bone marrow and spleen cells. Cell and Tissue Kinetics, 3(4), 393-403.

Hoffmann, M., Kuska, J.-P., Zscharnack, M., Loeffler, M., \& Galle, J. (2011). Spatial organization of mesenchymal stem cells in vitro-Results from a new individual cell-based model with podia. PLoS One, 6(7), e21960. https://doi.org/10.1371/journal.pone.0021960

Hope, K., \& Bhatia, M. (2011). Clonal interrogation of stem cells. Nature Methods, 8(4 Suppl), S36-S40. https://doi.org/10.1038/nmeth.1590

Jaccard, N., Szita, N., \& Griffin, L. D. (2017). Segmentation of phase contrast microscopy images based on multi-scale local basic image features histograms. Computer Methods in Biomechanics and Biomedical Engineering. Imaging \& Visualization, 5(5), 359-367. https://doi.org/ 10.1080/21681163.2015.1016243

Jonas. (2017, February 11). Plot spread points (beeswarm plot). MATLAB Central File Exchange from https://www.mathworks.com/ matlabcentral/fileexchange/37105-plot-spread-points--beeswarm-plot

MikeCF. (2014, March 6). Break Y axis. MATLAB Central File Exchange from https://www.mathworks.com/matlabcentral/fileexchange/ 45760-break-y-axis

Mollet, M., Godoy-Silva, R., Berdugo, C., \& Chalmers, J. J. (2008). Computer simulations of the energy dissipation rate in a fluorescence-activated cell sorter: Implications to cells. Biotechnology and Bioengineering, 100(2), 260-272. https://doi.org/10.1002/bit.21762

Muschler, G., \& Midura, R. (2002). Connective tissue progenitors: Practical concepts for clinical applications. Clinical Orthopaedics and Related Research, 395, 66-80. https://doi.org/10.1097/00003086200202000-00008

Muschler, G., Nitto, H., Boehm, C., \& Easley, K. A. (2001). Age- and genderrelated changes in the cellularity of human bone marrow and the prevalence of osteoblastic progenitors. Journal of Orthopaedic Research, 19, 117-125. https://doi.org/10.1016/S0736-0266(00)00010-3

Pérez-Silos, V., Camacho-Morales, A., \& Fuentes-Mera, L. (2016, 3187491). Mesenchymal stem cells subpopulations: Application for orthopedic regenerative medicine. Stem Cells International, 2016, -9. https://doi.org/10.1155/2016/3187491

Pittenger, M. F., Mackay, A. M., Beck, S. C., Jaiswal, R. K., Douglas, R., Mosca, J. D., ... Marshak, D. R. (1999). Multilineage potential of adult human mesenchymal stem cells. Science, 284(5411), 143-147. https://doi.org/10.1126/science.284.5411.143

Pochampally, R. (2008). Colony forming unit assays for MSCs. Methods in Molecular Biology, 449, 83-91. https://doi.org/10.1007/978-160327-169-1_6

Qadan, M. A., Piuzzi, N. S., Boehm, C., Bova, W., Moos, M. Jr., Midura, R. J., ... Muschler, G. F. (2018). Variation in primary and culture-expanded cells derived from connective tissue progenitors in human bone marrow space, bone trabecular surface and adipose tissue. Cytotherapy, 20(3), 343-360. https://doi.org/10.1016/j.jcyt.2017.11.013

Schindelin, J., Arganda-Carreras, I., Frise, E., Kaynig, V., Longair, M., Pietzsch, T., ... Cardona, A. (2012). Fiji: An open-source platform for biological-image analysis. Nature Methods, 9(7), 676-682. https://doi. org/10.1038/nmeth.2019

Schlie, S., Gruene, M., Dittmar, H., \& Chichkov, B. N. (2012). Dynamics of cell attachment: Adhesion time and force. Tissue Engineering. Part C, Methods, 18(9), 688-696. https://doi.org/10.1089/ten.TEC.2011.0635

Seiler, C., Gazdhar, A., Reyes, M., Benneker, L. M., Geiser, T., Siebenrock, K. A., \& Gantenbein-Ritter, B. (2014). Time-lapse microscopy and classification of 2D human mesenchymal stem cells based on cell shape picks 
up myogenic from osteogenic and adipogenic differentiation. Journal of Tissue Engineering and Regenerative Medicine, 8(9), 737-746. https:// doi.org/10.1002/term.1575

Siclari, V. A., Zhu, J., Akiyama, K., Liu, F., Zhang, X., Chandra, A., ... Qin, L. (2013). Mesenchymal progenitors residing close to the bone surface are functionally distinct from those in the central bone marrow. Bone, 53(2), 575-586. https://doi.org/10.1016/j.bone.2012.12.013

Villarruel, S. M., Boehm, C. A., Pennington, M., Bryan, J. A., Powell, K. A., \& Muschler, G. F. (2008). The effect of oxygen tension on the in vitro assay of human osteoblastic connective tissue progenitor cells. Journal of Orthopaedic Research, 26, 1390-1397. https://doi.org/10.1002/ jor.20666

Wang, D., Chen, R. U. I., Zhong, X., Fan, Y., Lai, W., \& Sun, X. (2014). Levels of $\mathrm{CD} 105^{+}$cells increase and cell proliferation decreases during $\mathrm{S}$ phase arrest of amniotic fluid cells in long-term culture. Experimental and Therapeutic Medicine, 8(5), 1604-1610. https://doi.org/10.3892/ etm.2014.1959

Zangle, T. A., \& Teitell, M. A. (2014). Live-cell mass profiling: An emerging approach in quantitative biophysics. Nature Methods, 11(12), 1221-1228. https://doi.org/10.1038/nmeth.3175

\section{SUPPORTING INFORMATION}

Additional supporting information may be found online in the Supporting Information section at the end of the article.

Data S1: Supporting Information

Figure S1: Caprine cells were used as a negative control for nonspecific binding of surface markers. Caprine bone marrow cells were harvested under a protocol approved by the Clevelend Clinic Institutional Animal Care and Use Committee. Mesenchymal stem cells were used as a positive contro; for CD73, CD90, and CD105 positive cells (Texas A\&M Health Science Center, Temple, Tx). Unstained trabecular surfce cells cells were used as a negative control for auto fluorescence and IgG1 Isotype control (Bio Legend, San jose CA) was used to test for nonspecific binding. Surface markers were stained at a 1:150 dilltion.
Antibodies demonstrated specific binding based on positive staining with MSCs used as a positive control. Antibodies demonstrated low non-specific binding based on low staining to caprine cells and low isotype staining to trabecular surface cells. All scale bars $500 \mu \mathrm{m}$.

How to cite this article: Kwee E, Saidel G, Powell K, Heylman C, Boehm C, Muschler G. Quantifying proliferative and surface marker heterogeneity in colony-founding connective tissue progenitors and their progeny using time-lapse microscopy. J Tissue Eng Regen Med. 2019;13:203-216. https://doi.org/ 10.1002/term.2782

\section{APPENDIX A}

\section{IMAGE PROCESSING AND ANALYSIS}

Image processing and analysis was performed using MATLAB and Fiji using the following workflow. Phase-contrast processing, cell segmentation, and colony segmentation were performed using custom MATLAB scripts. Cell marker segmentation was performed sequentially using Fiji. All time points were processed for phasecontrast processing, cell segmentation, and cell marker segmentation. Day 6 images were processed using automated colony segmentation. Based on the resulting output masks from MATLAB and Fiji processing, analysis of colony parameters at Day 6 was performed in Fiji using the Analyze Particles function on the Colony Mask and redirecting to the Cell Mask and Cell Marker Masks to get density metrics.

For all other time points, cells were manually traced back to identify the colony lineage and the colony-founding cell. Image registration between tiles is not critical for cell modelling as model inputs are made
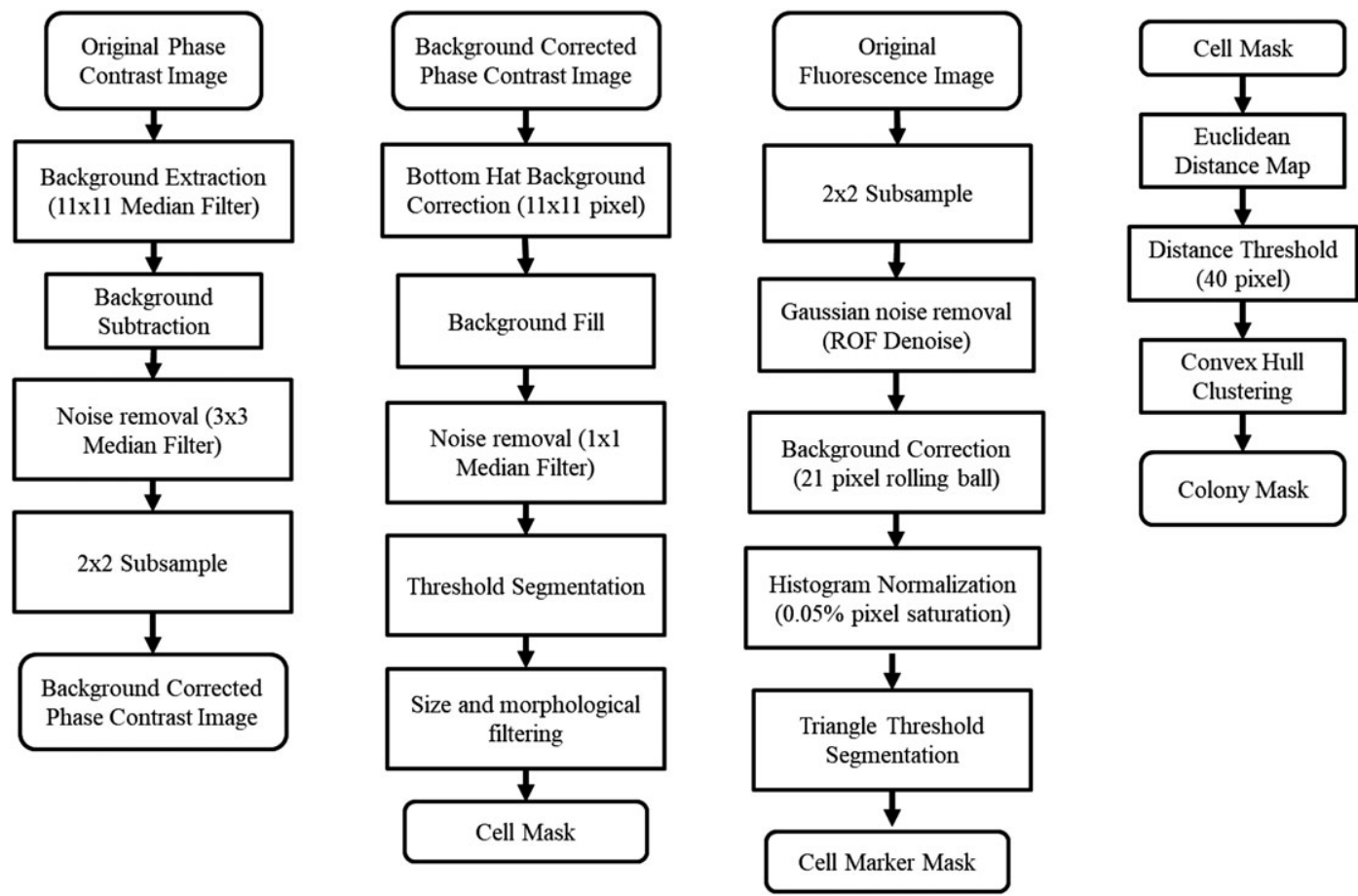

FIGURE A1 Flow chart of image processing and analysis steps. ROF: Rudin-Osher-Fatemi 
with respect to the centroid of the colony, which is automatically identified. Image registration can be added to improve visualization for identifying the colony-founding cell.
On the basis of cell tracking and resulting masks, analysis of fluorescence images at 24-hr intervals was similarly processed in Fiji using the Analyze Particles function on the Colony Mask and redirecting to
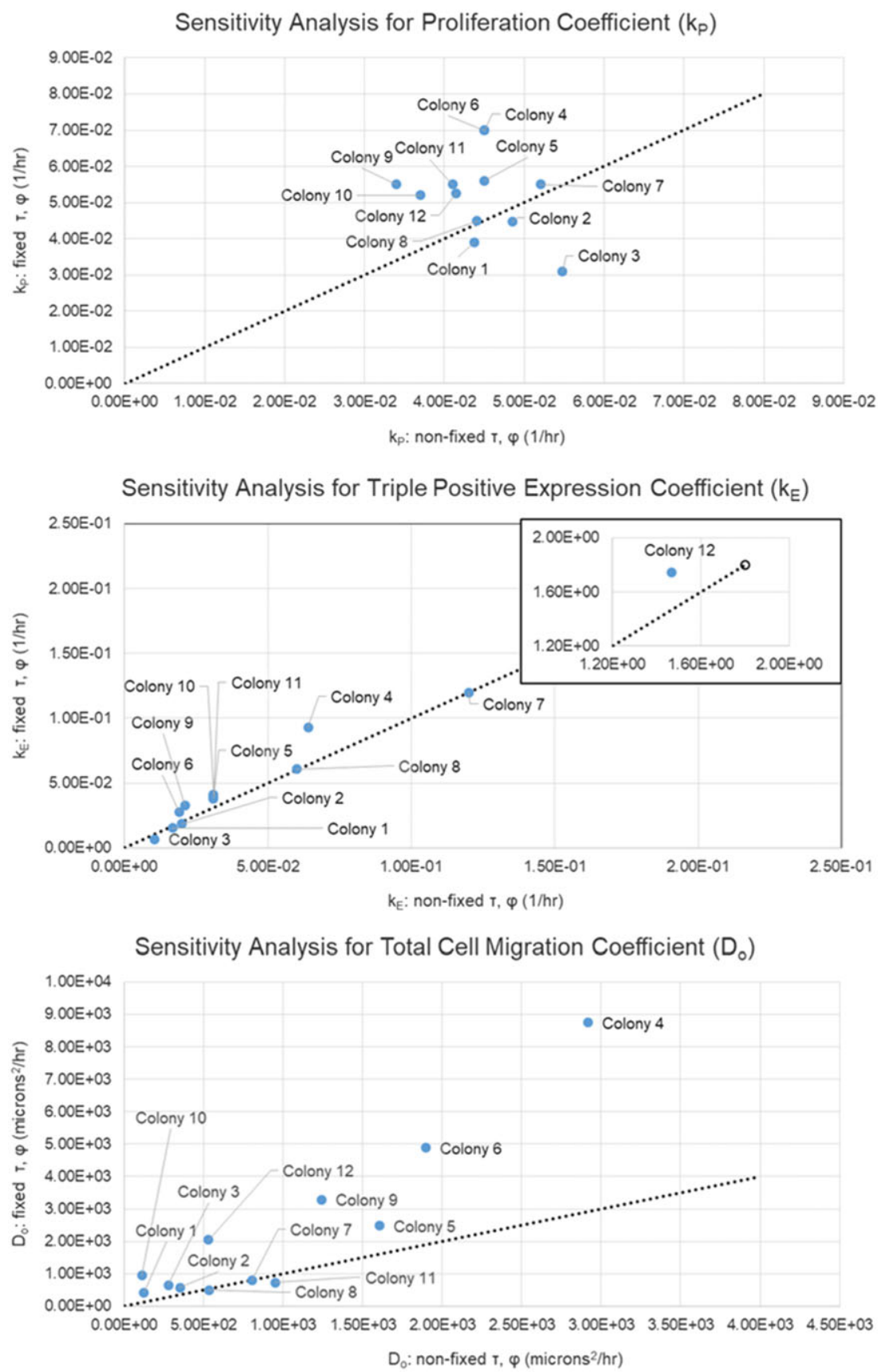

FIGURE B1 Sensitivity analysis for colony parameters. Parameters were estimated for nonfixed and fixed cases for lag phase and contact inhibition. Dotted line in each plots represents 1:1 line where points would lie when parameters were unchanged [Colour figure can be viewed at wileyonlinelibrary. com]

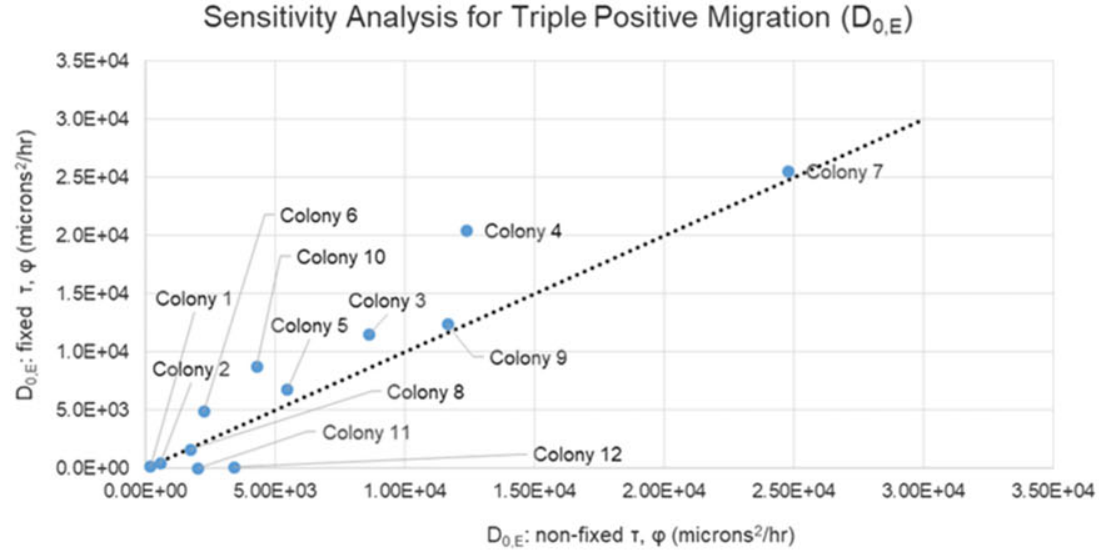


the Cell Mask and Cell Marker Masks to get density metrics. Cell Masks, Cell Marker Masks, and Colony Masks from all time points were used as inputs into the colony model.

Plots made in MATLAB using modified beeswarm plot and break axis functions from MATLAB Central File Exchange (Jonas, 2017; MikeCF, 2014). MATLAB source code for image processing analysis and colony model parameter estimation is available at https://sites. google.com/case.edu/edward-kwee/.

\section{APPENDIX B}

\section{COLONY PARAMETER SENSITIVITY ANALYSIS}

Sensitivity analysis was performed by examining simulations with fixed lag phase duration $(\tau)$ at $40 \mathrm{hr}$ and contact inhibition constant $(\varphi)$ at 2 . These values were chosen to analyse colony formation after most colonies passed the lag phase. Most colonies initiated proliferation within $40 \mathrm{hr}$ of the lag phase. A contact inhibition constant of 2 was within the range of estimated parameter values. From simulations, the effect of parameter change was evaluated, and colonies were reranked.

When fixing lag phase and contact inhibition, proliferation rates were largely unchanged between nonfixed and fixed parameter simulations. The estimates were of the same order of magnitude in both simulation cases (Figure B1). Parameter estimates for total cell proliferation, triple positive cell proliferation, and triple positive cell migration were relatively unchanged with parameters lying close to or equally distributed across the 1:1 line. For fixed lag phase and contact inhibition, estimates of the total cell migration coefficient were higher than for the nonfixed case. This can be attributed to migration occurring before the $40 \mathrm{hr}$. This indicates that early cell contact is important for accurate parameter estimation. Overall, the model parameters were comparable, within one order of magnitude when lag time and contact inhibition were not fixed.

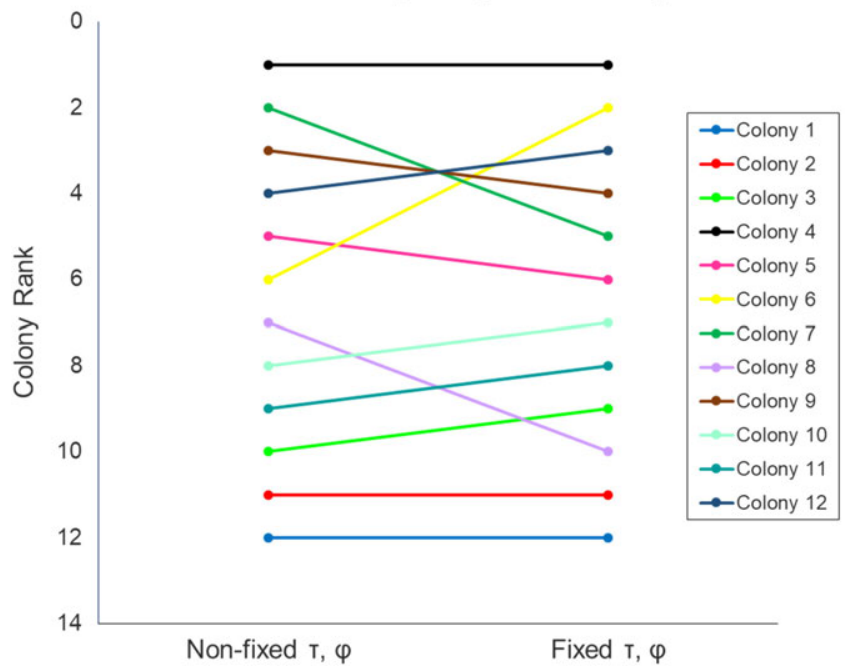

FIGURE B2 Sensitivity analysis for colony rank. Colony rank was evaluated following parameter estimation with fixed lag phase and contact inhibition constant. Three colonies had ranks change by one than one rank. The remaining nine colony ranks were unchanged or changed by one [Colour figure can be viewed at wileyonlinelibrary. com]

Additionally, when reranking colonies desirable for use as a therapy, we found that the colony ranks were mostly unchanged (Figure B2). With the exception of three colonies, colony rank was either unchanged or changed by only one position. The highest ranked colony was also unchanged. Therefore, given that parameter estimates were within the same of order of magnitude and colonies' ranks were generally unchanged, it appears that the model analysis is insensitive to changes in lag phase and contact inhibition parameters. 